\title{
The ATLAS Liquid Argon Electromagnetic Calorimeter: Construction, Commissioning and Selected Test Beam Results
}

\author{
Luis Hervas and The ATLAS Liquid Argon Electromagnetic Calorimeter Group
}

\begin{abstract}
The construction of the ATLAS liquid argon electromagnetic calorimeter has been completed, and commissioning is in progress to prepare the cryostats for lowering into the ATLAS pit. After a brief description of the detector, its construction, and readout electronics, this paper summarizes results of quality checks (electrical and connectivity) carried out during the integration of the calorimeter wheels into the cryostats. This paper also presents selected results of its performance, such as linearity, energy resolution, timing resolution, and uniformity of the energy response, obtained in beam tests with several series modules.
\end{abstract}

Index Terms-Calibration, calorimeters, liquid argon, particle physics.

\section{INTRODUCTION}

$\mathbf{T}$ HE ATLAS detector, currently under construction, envisaged to operate at LHC, CERN's future proton-proton collider in Geneva, has chosen the lead-liquid argon (LAr) sampling technique with accordion geometry for its electromagnetic calorimetry. The detector has to cope among other challenges with a particle bunch-crossing frequency of $40 \mathrm{MHz}$ and a dynamic range spanning six orders of magnitude, from a few mega electronvolts to several tera electronvolts energy depositions. The electromagnetic calorimeter will play a crucial role in the precise detection of electrons and photons, jets, and missing energy measurements in final states of processes which are expected to be observed and studied at LHC, for example $H \rightarrow \gamma \gamma, H \rightarrow 4 e$, and $H \rightarrow W W$.

The required performance (see [1] and [2]) from the apparatus, determined from simulations of physics processes like those previously described includes the following:

- an energy resolution lower than $10 \% / \sqrt{E(\mathrm{GeV})}$ with a constant term smaller than $1 \%$, combined with a linearity of the energy response better than $1 \%$ up to the high energy domain;

- $\quad$ full solid angle coverage down to $\eta=3.2$;

- a timing resolution below 100 ps for high energy deposits;

- angular resolution smaller than $50 \mathrm{mrad} / \sqrt{E(\mathrm{GeV})}$.

The liquid argon technique provides good linear behavior, long term stability, and radiation tolerance. With the accordion geometry, a "hermetic" detector can be achieved. It also minimizes inductances in the signal paths allowing a fast signal shaping

Manuscript received June 15, 2004; revised April 8, 2005.

The author is with the Physics Department, CERN, CH-1211 Geneva 23, Switzerland (e-mail: Luis.Hervas@cern.ch).

Digital Object Identifier 10.1109/TIM.2005.851233 which is needed to cope with the 25-ns bunch-crossing intervals.

This paper covers briefly the detector design, its construction, including commissioning tests during integration, a short description of the readout electronics, and performance results from test beam measurements performed on individual modules.

\section{DETECTOR DESCRIPTION}

The calorimeter is divided in two half-barrel wheels (sides $\mathrm{H}$ and I) covering the pseudorapidity up to $|\eta| \leq 1.475$, housed in the barrel cryostat and two end-cap detectors $(1.375 \leq|\eta| \leq$ 3.2) in two end-cap cryostats (EC-A and EC-C).

The stack making up the calorimeter is composed of three elements.

1) Absorbers: The absorbers (made of lead glued between thin stainless steel sheets) are 2.2-mm-thick plates that are formed to accordion shape. The edges are glued with G10 bars for mechanical support.

2) Electrodes: The readout circuits are made of three copper layers insulated by two layers of polyimide. The two outer layers, split in sectors, are connected to high-voltage (HV) sources and polarize the LAr gap to the absorber. The inner layer is where the signal is collected through capacitive coupling and is segmented in readout pads.

3) Spacers: The spacers made of honeycomb-structured NOMEX paper impregnated with phenolic resin keep the electrodes centered in the gap between two absorbers.

Structural elements are needed to keep the absorbers in place. In the case of the barrel, where the G10 bars are in contact with each other and precisely define the LAr gap, stainless steel rings at the outer and thin G10 rings at the inner calorimeter radii rigidify the cylinder. The end-cap geometry is given by rings (composite material or aluminum) with index holes that take pins locating the position of the absorber G10 bars.

The main difference between the barrel and end-cap is the gap geometry.

- Barrel: In the barrel, the LAr gap can be kept at constant (2.1-mm half-gap) thickness from front to back by opening the folds of the accordion. The HV value is the same for all gaps $(2 \mathrm{kV})$.

- End-cap: The end-cap geometry opens the gap at increasing radius. For mechanical reasons, two concentric 


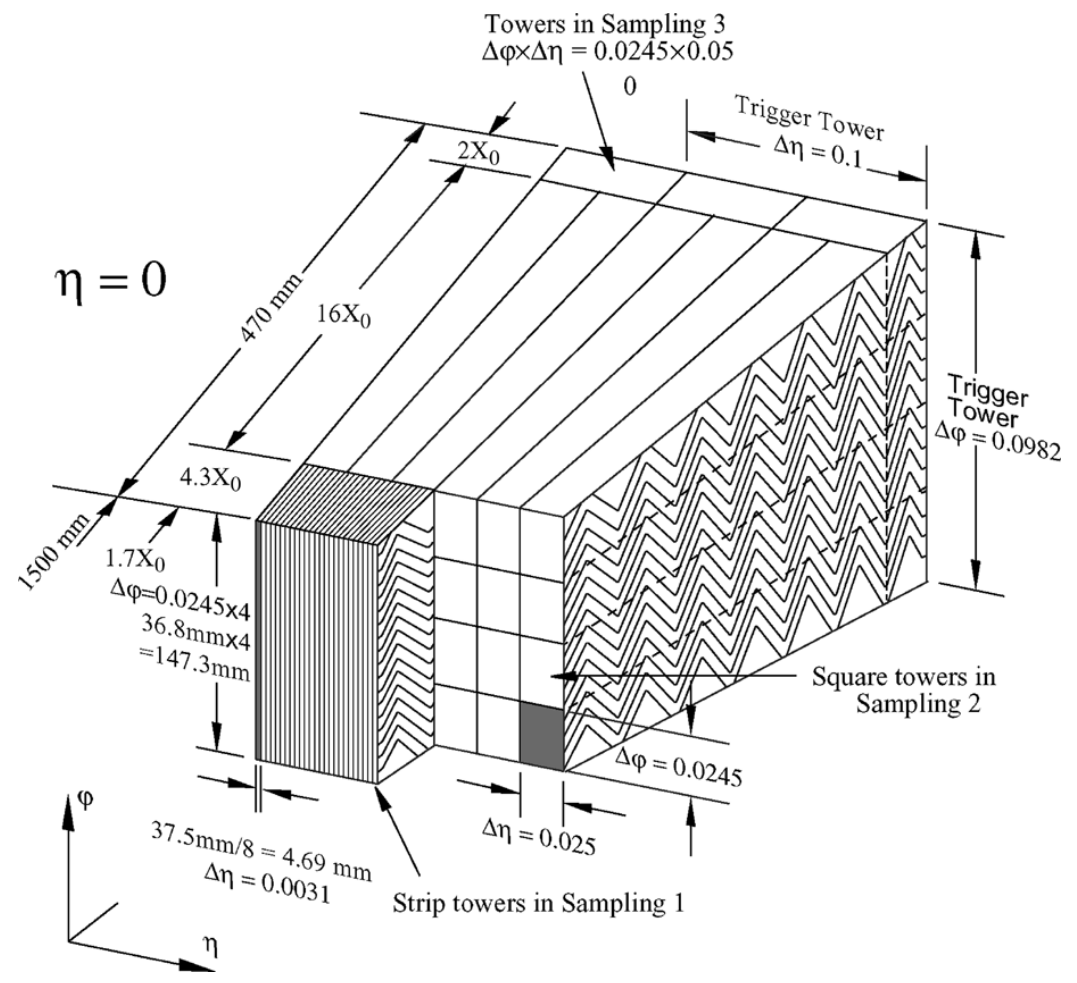

Fig. 1. Segmentation of the electromagnetic (EM) barrel calorimeter.

(a "big" outer and a "small" inner) wheels have half-gaps varying from 3.1 to $0.9 \mathrm{~mm}$. This requires varying HV to keep the detector response independent of $\eta$. In a practical way, the $\mathrm{HV}$ has nine steps from 0.9 to $2.5 \mathrm{kV}$.

The charge deposited in the LAr gaps is collected on the cells of the electrodes. The $\eta$ granularity and the depth separations are made by divisions etched on the copper surface of the circuit. Readout cells are made by summing signals of a certain number of gaps directly at the back or front of the modules (see Fig. 1) and bringing these out via feedthrough connections to readout electronics placed directly on the cryostats.

Except at several edge zones (e.g., the interface zone between the barrel and end-caps modules), the segmentation of the calorimeter presents three compartments in depth:

1) the first sampling (front, S1), optimized for $\gamma / \pi^{0}$ separation, ends at $6 X_{0}$, including $\sim 1.6 X_{0}$ of dead matter in front of the active part of the calorimeter, and has a granularity of $\Delta \eta \times \Delta \phi \simeq 0.025 / 8 \times 0.1)$;

2) the second sampling (middle, S2) with a depth of 16 to $18 X_{0}$ contains most of the $e / \gamma$ energy and has a granularity of $\Delta \eta \times \Delta \phi \simeq 0.025 \times 0.025$;

3 ) the third sampling (back, S3) covers the remaining depth $\geq 2 X_{0}$ and has a granularity of $\Delta \eta \times \Delta \phi \simeq 0.05 \times 0.025$ to help reconstruct high-energy showers and discern between hadronic and electromagnetic showers.

In the range $|\eta| \leq 1.8$ a thin presampler (PS) detector is used to correct for energy losses in "dead material" in front of the calorimeter. The total number of readout channels is more than 170000 (173312 total: 9344 in PS, 85760 in S1, 52096 in S2, and 26112 in S3).

\section{Detector CONSTRUCTION}

The construction plans of the LAr calorimeter are described in detail in [3]. In addition to the electromagnetic part relevant for this paper, the description includes the hadronic end-cap (HEC) and the forward (FCAL) calorimeters, which share the liquid argon in the same cryostat vessels.

The series modules were constructed between 2001 and 2003. This was the conclusion of a long research and development process started in 1990 [4]-[7]. The performance of a LAr calorimeter for an LHC experiment was demonstrated with a "2-m" (long) prototype of the RD-3 Collaboration [8]. The technique for building the ATLAS EM-calorimeter was further refined with the construction and beam tests of "Module 0 's," which used elements from (or close to) series production. These modules were thoroughly tested in test beams [9], [10].

The series modules stacked at several collaborating institutes were integrated into "wheels" at CERN and inserted into their corresponding cryostats. At the time of this paper's conference presentation, the barrel wheels (Fig. 2) are inserted, and the barrel cryostat is closed (welded), and one of the end-cap wheels is inserted in its cryostat (Fig. 3). At the time of the paper's submission for publication, the barrel cryostat has been lowered in the ATLAS pit after cold-testing in the surface, though not yet placed in its final position; one end-cap cryostat has been cold-tested and is in the process of warming up, while the second end-cap cryostat has been closed.

In order to comply with the performance requirements mentioned previously, especially the constant term of the energy resolution, a strict quality control at construction time is mandatory [11]. We show some examples of QC for different parts and steps. 


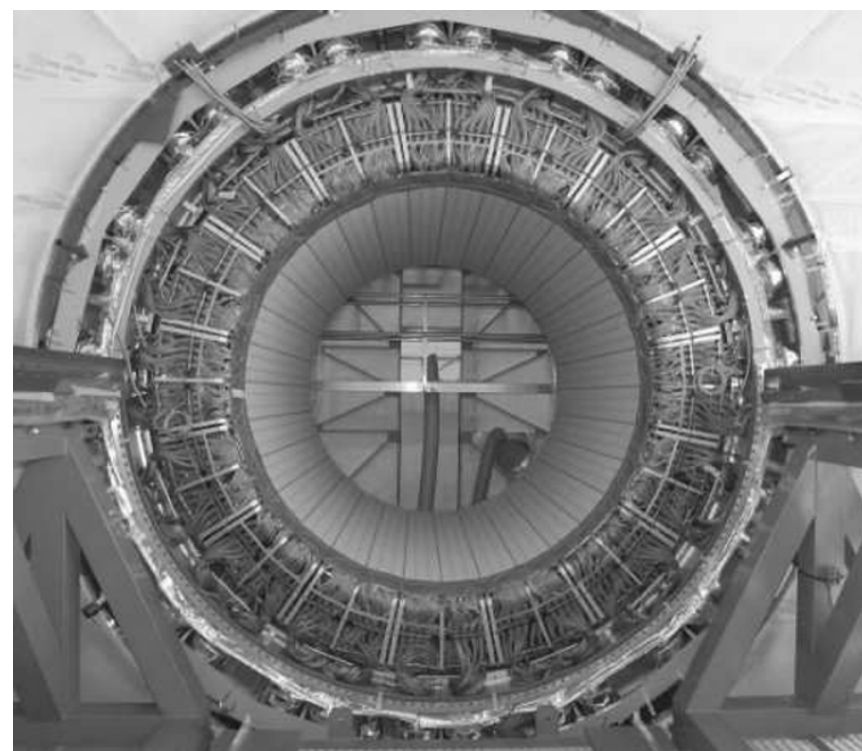

Fig. 2. View of a barrel wheel after insertion into the cryostat.

- Lead: Local variations of lead thickness lead to changes in the calorimeter response (raw lead thickness controlled to $\pm 7 \mu \mathrm{m})$. Measuring the thickness of each plate with an ultrasound system [12] and grouping absorbers in an "intelligent" way, the constant term contribution of this point can be limited to $0.1 \%$.

- G10 bars: The precise machining of the wedge shape of the barrel G10 bars determines the final geometrical accuracy of the cylinders.

- Electrodes: The position resolution of the detector is a direct consequence of the precise etching and positioning of the electrodes on the absorbers [13].

- Stacking: The stack of absorbers-spacers-electrodes is controlled during stacking and shimmed at the bars $(50 \mu \mathrm{m})$ if necessary.

- Gaps: The uniformity of gaps is checked by measuring their capacitances in several sector subdivisions.

- $H V: \mathrm{HV}$ tests are performed all along the stacking and assembly process.

- Cabling: Various cabling tests (connectivity, impedance control) are needed to insure good electrical connections at all steps of the electronics chain.

- Cryogenic tests: All modules undergo a test at cryogenic temperatures. At this point, $\mathrm{HV}$ and signal connectivity tests are routinely performed.

Quite a few tests are carried out during the construction steps of the calorimeter modules. Final tests at integration time of the single modules into the wheels include the following.

- Test pulse injection sends a fast step signal through the calibration lines and reads the output through the readout lines where a signal is expected.

- The electrode connectivity and capacitance test is a test in which a "low" frequency $(1-20 \mathrm{~Hz})$ signal is injected at the HV lines and generates by capacitive coupling a signal on the readout lines corresponding to that HV area.

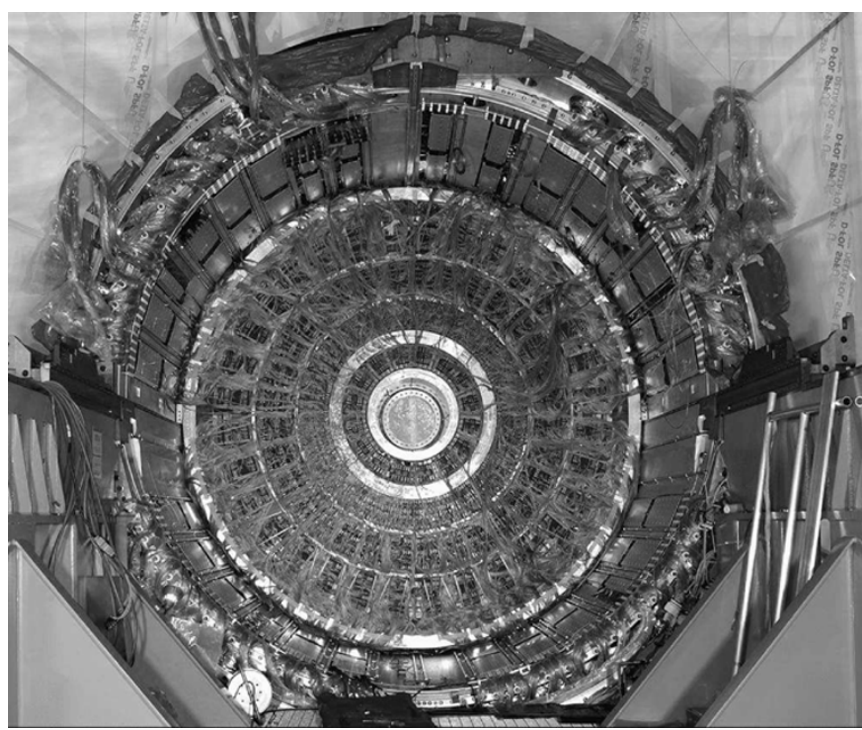

Fig. 3. View of an end-cap wheel after insertion into the cryostat but before the hadronic wheels are pushed behind.

- HV tests verify the integrity of the HV connections (looking at the capacitive loading current) and the HV "holding" of the gaps.

- Verification measurements of the individual calibration resistors. This operation can be performed directly from the baseplane circuit at the exit of the feedthroughs.

- LC measurements determine the precise inductance and capacitance of a readout cell. This measurement is done with a network analyzer, injecting signal into the calibration input and looking at the resonance frequency of the signal coming via the readout line. Fig. 4 (top) shows a typical transfer function for a middle cell. The resonant frequency $\nu_{0}$ can be well identified. The measured values for $\nu_{0}$ of all middle cells in one half-barrel wheel as a function of $\phi$ are plotted in Fig. 4 (bottom). Four groups have been formed from $\eta<0.4$ to $1.2<\eta<1.4$. In each one, the mean resonance frequency has been computed. We observe as expected decreasing $\nu_{0}$ with increasing pseudorapidity coming from larger cell capacitance and/or inductance. Inside each region, the observed small structure with $\phi$ agrees with channel-to-channel inductance variations originated in the summing boards.

The final test before transporting the cryostats into the ATLAS pit is a full cool-down and LAr filling. At this point, full HV tests are performed, and the electrical tests are repeated. At the time of these proceedings, this cool-down is being prepared. At warm, after insertion in the cryostat, the number of faulty readout channels for the whole barrel and one end-cap calorimeter is shown in Table I. The last column shows the number of calibration resistors outside the $\pm 0.2 \%$ tolerance limit (results only for one half-barrel and one end-cap wheel).

All modules have undergone cryogenic qualification, including "long" (days) HV tests. The gaps and sectors showing a problem were cabled separately to additional HV sources. While no whole-wheel tests in LAr have been done at this point, $\mathrm{HV}$ tests in air at $1400 \mathrm{~V}$ were repeatedly performed during the 

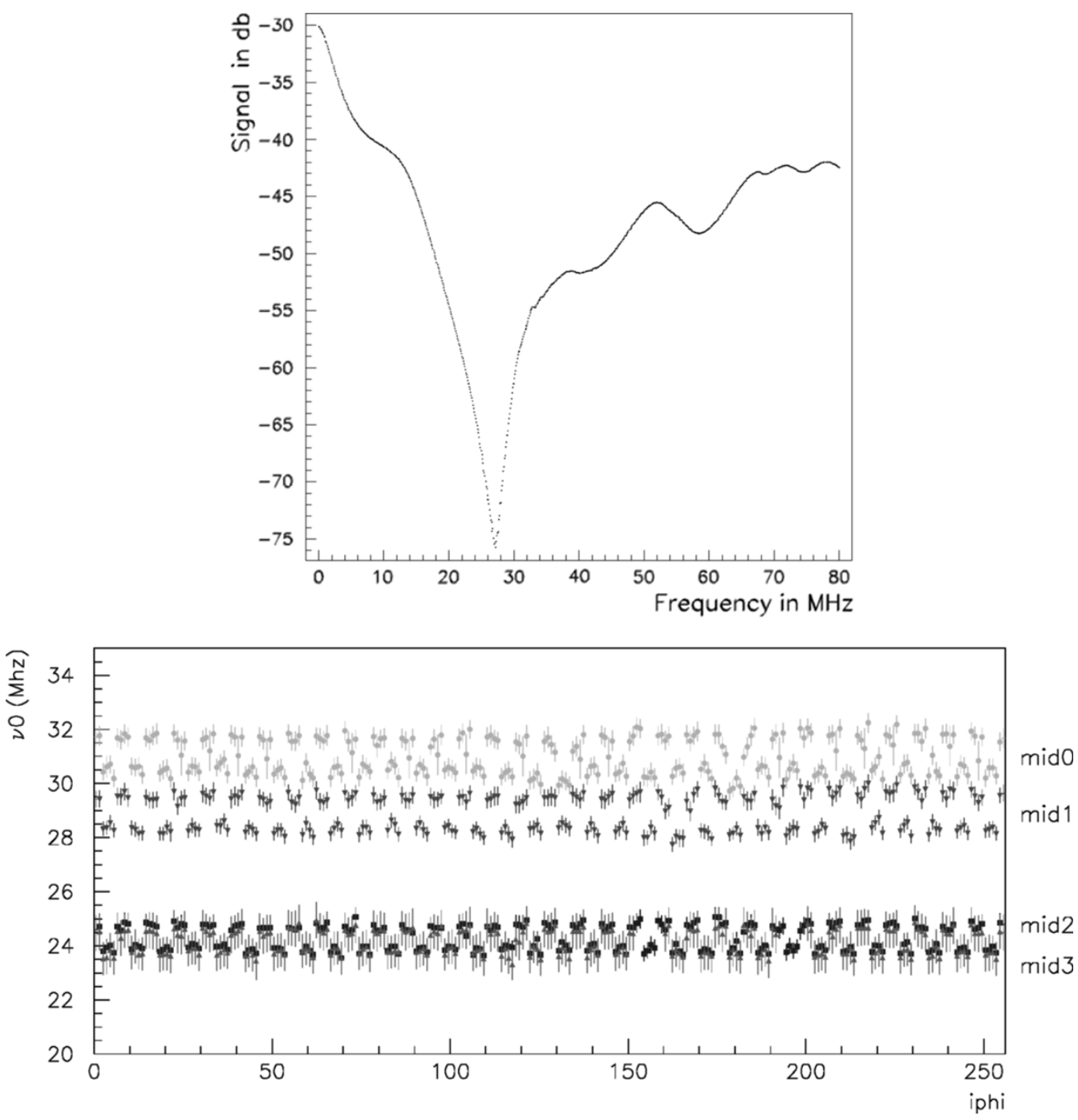

Fig. 4. Top: Network analyzer output signal amplitude for a middle cell. Bottom: Resonant frequency for all middle cells around one half-barrel wheel (256 units of $\phi$ ) grouped in four pseudorapidity regions: mid0: $\eta<0.4$, mid1: $0.4<\eta<0.8$, mid2: $0.8<\eta<1.2$, and $\operatorname{mid} 3: 1.2<\eta<1.4$.

TABLE I

Count of Defective Calorimeter Readout Channels AND CALIBRATION RESISTORS FOUND AFTER INSERTION OF THE WheEls IN the CRYostat. No CRYOGENIC TeSt Yet. TOP: BARREL WhEELS, BOTTOM: ONE END-CAP WHEEL (EC-C)

\begin{tabular}{|c|c|c|c|c|c|}
\hline$\overline{\text { Barrel }}$ & Total\# & Maximum & \multicolumn{2}{|c|}{ "E\# channels } & \multirow{2}{*}{$\begin{array}{l}\text { Calibration } \\
\text { resistors }\end{array}$} \\
\hline Layer & channels & acceptable & $\max$ & found & \\
\hline presampler & 7808 & $0.05 \%$ & 4 & 0 & $5(0.1 \%)$ \\
\hline strips & 57216 & $2 /$ mod. & 64 & 9 & $16(0.05 \%)$ \\
\hline middle & 28672 & $0.05 \%$ & 14 & 2 & $13(0.07 \%)$ \\
\hline back & 13824 & $0.05 \%$ & 6 & 0 & $12(0.13 \%)$ \\
\hline barrel-end & 2048 & $0.05 \%$ & 1 & 3 & $4(0.05 \%)$ \\
\hline calibration & 8192 & $0.05 \%$ & 4 & 1 & \\
\hline \multicolumn{6}{|l|}{$\overline{\text { EC-C Layer }}$} \\
\hline presampler & 768 & $0.05 \%$ & 0 & 0 & 0 \\
\hline strips & 14272 & $2 / \bmod$. & 16 & 2 & $8(0.05 \%)$ \\
\hline middle & 11712 & $0.05 \%$ & 6 & 1 & $12(0.10 \%)$ \\
\hline back & 5120 & $0.05 \%$ & 3 & 0 & $1(0.02 \%)$ \\
\hline calibration & 2976 & $0.05 \%$ & 2 & 0 & \\
\hline
\end{tabular}

assembly of the wheels and their insertion. In the barrel, out of 14336 electrode sectors, $21(0.15 \%)$ are cabled separately due to a potential problem. Out of a total of 448, $21 \mathrm{HV}$ sectors (representing $2.3 \%$ of the acceptance) have been split to two HV sources since they showed a problem during the cold test, which could not be traced to a particular gap. We should note that $\mathrm{HV}$ problems show up in one of the half-gaps. Reducing (or disconnecting) the $\mathrm{HV}$ on one side of the electrode is a "simple" geometrical effect that has been well studied in the test beams and can be easily corrected. Up to now, none of the calorimeter detection volume is "dead" due to HV faults.

\section{READOUT ELECTRONICS}

The main requirements that the detector and its performance set upon the readout electronics for the calorimeter includes following main points:

- dynamic range equivalent to $16 \mathrm{~b}$;

- calibration precision such that its contribution to the energy resolution constant term is kept below $0.25 \%$;

- coherent noise per channel below $5 \%$ of the total noise; 


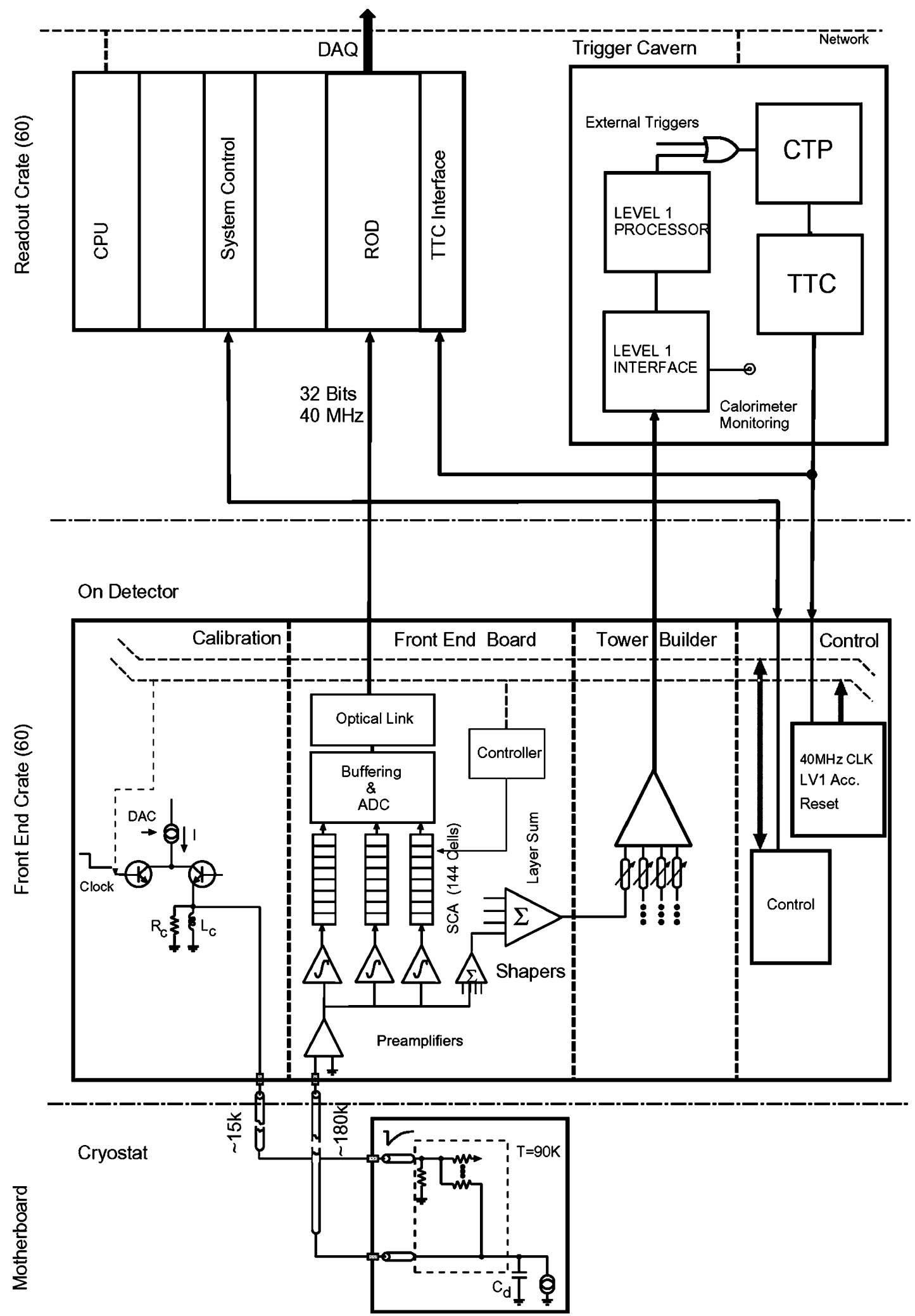

Fig. 5. Calorimeter readout scheme. Three parts are shown from bottom to top: inside the cryostat, on the cryostat, and in the (remote) counting room.

- accept signals at the bunch-crossing frequency of $40 \mathrm{MHz}$;

- $\quad$ store signal samples during up to $2.5 \mu$ s of the level 1 trigger latency;

- provide dead-timeless operation at the L1 maximum trigger rate of $75 \mathrm{KHz}$;
- make energy sums for trigger towers at a granularity level of $\Delta \eta \times \Delta \phi=0.1 \times 0.1$;

- tolerate (with safety factors) "moderate" radiation levels of $5 \mathrm{kRad}$ of ionizing radiation, $1.610^{12} \mathrm{n} / \mathrm{cm}^{2}$, and $7.710^{11}$ hadrons $/ \mathrm{cm}^{2}$ with energy over $20 \mathrm{MeV}$, for ten years at LHC; 
- high reliability due to limited access when in ATLAS;

- high density and low power consumption (0.8 W/chan) given the high number of channels $(170 \mathrm{~K})$ required.

The design of the electronics readout is shown in Fig. 5 and is described in detail in [3] and [14].

Inside the cryostat, we have only the precision calibration and termination resistors (no active components, the so-called $\emptyset T$ configuration) placed on "motherboards."

Directly placed at the feedthroughs, the front end crates (FEC) house large PCBs $(400 \mathrm{~mm} \times 500 \mathrm{~mm})$. A calibration board injects fast triangular pulses down the calibration lines. Pulses need to be very precise demanding a very good uniformity $(0.2 \%)$ and low crosstalk $(\sim 0.1 \%)$. Readout signals are received in the front end board (FEB) which incorporates the following functions:

1) signal amplification by bipolar preamplifier hybrid circuits;

2) signal shaping (bipolar $\mathrm{CR}-\mathrm{RC}^{2}$ integrated circuits) with a time constant of $15 \mathrm{~ns}$ required to clip the readout current within the bunch-crossing interval;

3) three-way gain split (gains of 1,10 , and 100) needed to cover the large dynamic range;

4) analog summing over cells in the same layer;

5) 40-MHz sampling of signals and storage in an analog pipeline [switched capacitor array (SCA)] during the L1 trigger latency;

6) digitization (12 b@5 MHz) of typically five samples of the relevant gain scale (to be selected on the fly);

7) data multiplexing formatting and bit-serial transmission of the information of 128 channels via one optical link operated at $1.6 \mathrm{~Gb} / \mathrm{s}$ to off-detector electronics.

Fig. 6 shows one FEB board. The enumerated functions can be seen from bottom to top in the board flow. Each side of the board handles half of the 128 readout channels. The system is completely data-driven with all the control information being added to the data stream. The implementation of most of the functions is done with application-specific integrated circuits (ASICs) manufactured in various radiation-hard technologies [e.g., Durci Mixte sur Isolant Logico-Lineaire (DMILL), Deep Sub Micron (DSM)]. Several parameters to the ASICs can be downloaded at initialization time allowing certain flexibility needed to cope with different beam conditions or trigger strategies.

Other boards in the FECs are the controller board needed to distribute the timing and trigger signals (TTCs) as well as control data to all other boards and the tower builder board, which further adds the trigger signals and drives them off-detector to the trigger receiver and trigger processing electronics. The boards are covered by aluminum plates connected to an underpressured water cooling system.

The third part of the electronics is located in the counting room, down 75-100 m of cables, off the detector. In addition to the trigger receiver electronics and the TTC distribution system, the main readout is covered by the readout driver (ROD) boards. There, the optical link fibers are received, and the digitized samples are processed by digital signal processors (DSPs). Digital

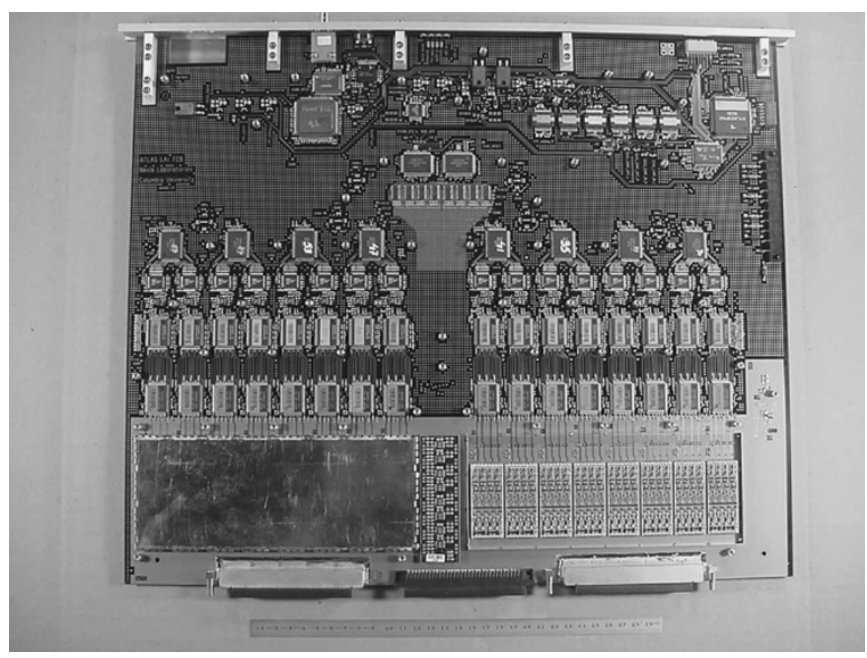

Fig. 6. Readout electronics FEB.

filtering algorithms are applied in the form of linear combination of samples weighted with coefficients determined through an "optimal filtering" technique (see [15]). By adequately choosing these, the signal amplitude (energy) and time (delay relative to the sampling clock) can be reconstructed in a way to minimize the noise. The optimal filtering coefficients are established from the noise autocorrelation matrix of the samples and the pulse shape and its derivative. These are obtained from calibration runs, but in order to apply them to physics pulses, subtle effects like the inductance between the calorimeter cells (where the physics signal is created) and the motherboards (where the calibration signal is injected) have to be taken into account.

\section{Performance Results From Test Beam}

A total of four barrel and three end-cap calorimeter modules (in addition to the "Module 0's") were exposed to muons and electrons beams with energies up to $245 \mathrm{GeV}$ on the $\mathrm{H} 8$ and $\mathrm{H} 6$ beamlines of CERN's Super Proton Synchrotron (SPS). They were read out with front-end electronics similar to the one described previously (albeit non-radiation hardened version). The results of these tests validated both the detector construction as well as the electronics design. As illustration, we show a selection of these results.

- Muon response: The calorimeters response to "minimum ionizing" particles was studied [9] with muons contained in the electron beam. A cluster of two $\phi$ cells is used in this case. The deposited energy (with energy scale calibrated from electrons) can be seen in Fig. 7, which also shows the energy of "random" events. The ratio of the most probable muon energy to the root mean square (rms) of the noise distribution is the signal-to-noise ratio (7.11 \pm 0.07 ). With muons, a $0.4 \%$ module uniformity is found. For such small signals, best results are only obtained by applying an optimal filtering algorithm with the corrections mentioned previously.

- Energy resolution: The energy resolution is measured from energy scan performed on several calorimeter cells. It can be fit with the function $\sigma_{E} / E=a / \sqrt{E} \oplus b$. After unfolding the noise and beam energy uncertainty from 


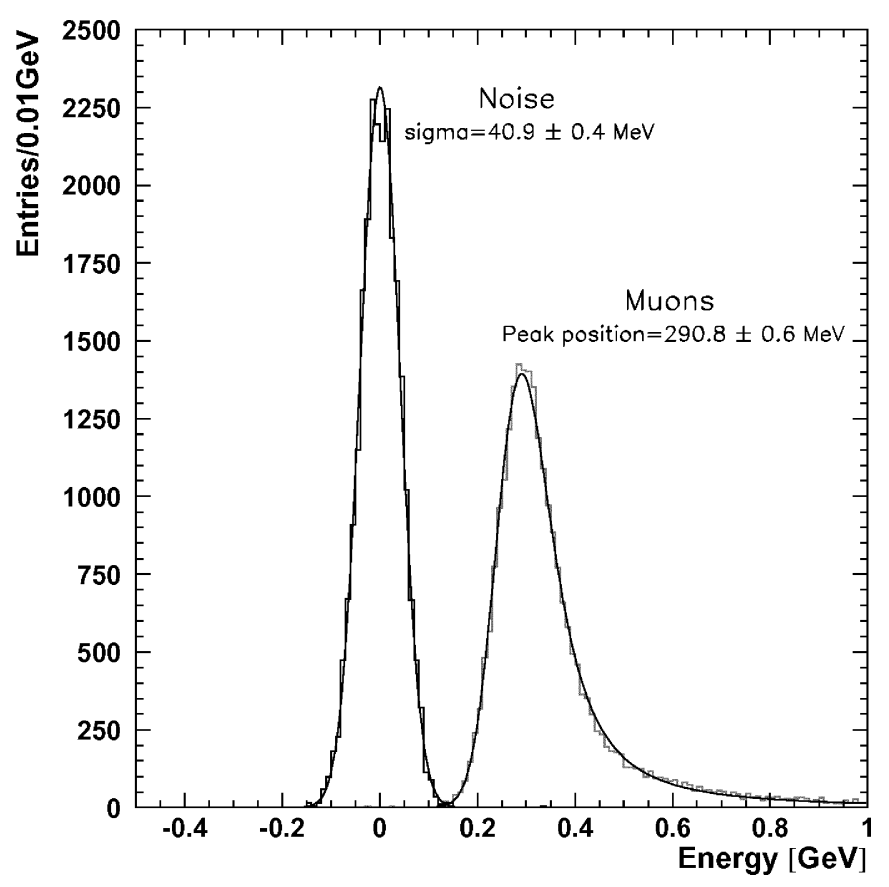

Fig. 7. Muon signal in a $1 \eta \times 2 \phi$ middle cluster and noise signal for "random" events.

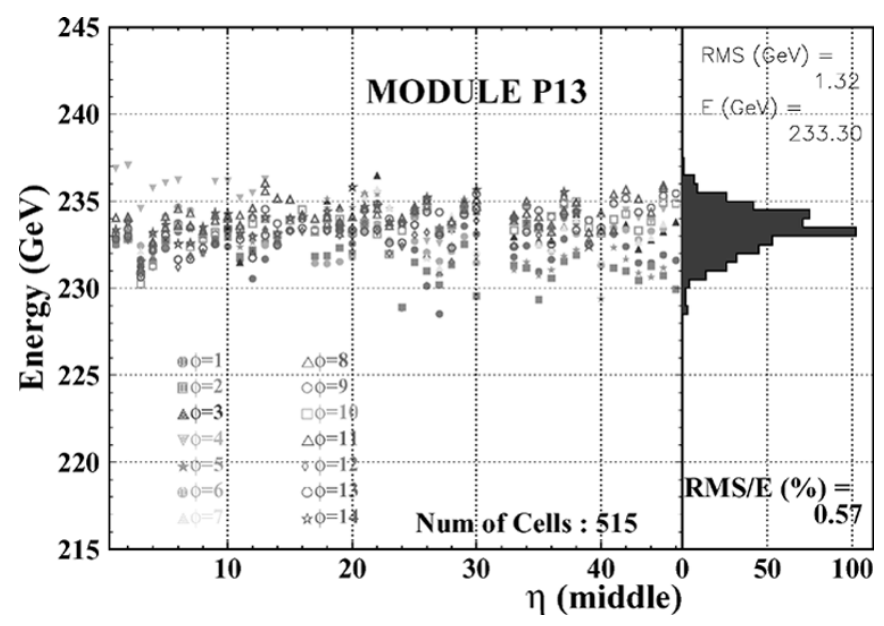

Fig. 8. Energy response and uniformity for barrel module P13 with electrons of $232 \mathrm{GeV}$.

the energy spectrum, the sampling term $a$ measured for barrel (end-cap) modules is less than 9.5\% (12.5\%) and the local constant term $b$ less than $0.3 \%(0.5 \%)$.

- Response uniformity: The calorimeter uniformity energy response has been systematically measured with beam scans in each cell along $\eta$ and $\phi$. For this reconstruction, energy is summed in a cluster of $3 \times 3$ cells of the middle compartment around the most energetic one $(\Delta \eta \times \Delta \phi=$ $0.075 \times 0.075)$. The nonuniformity is found to be below $0.6 \%$ for a full module as shown in Fig. 8 for a barrel module. For local regions of $\Delta \eta \times \Delta \phi=0.2 \times 0.4$, it is less than $0.5 \%$. Very similar numbers are obtained for end-cap modules.

- Linearity: A special effort was made to precisely measure the beam energy (arriving to a few $10^{-4}$ ) in dedicated

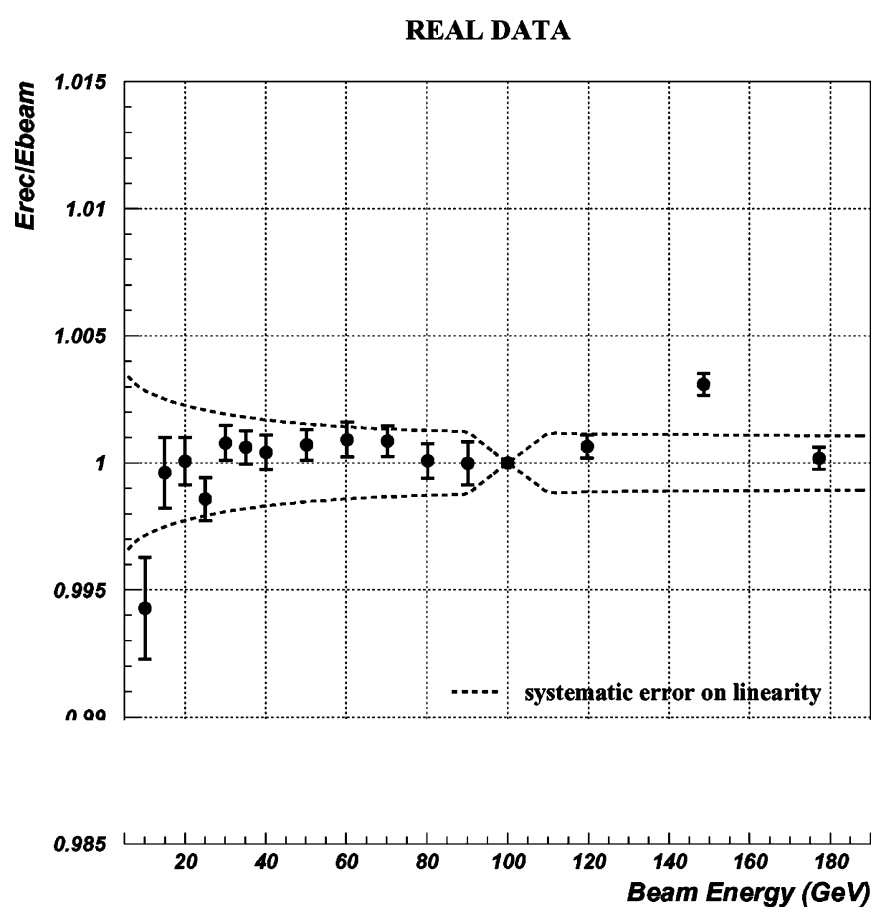

Fig. 9. Nonlinearity response for electrons.

linearity studies in the range $10-180 \mathrm{GeV}$ [16]. With corrections for factors like magnet currents, magnetic probe temperature dependence plus a smart weighting of the presampler signal and corrections for energy leakage (with the help of Geant 4 simulated data), the nonlinearity observed is at the level of $0.2 \%$ (see Fig. 9).

- Timing resolution: The optimal filtering algorithm can reconstruct the time of the energy depositions relative to the sampling clock. In ATLAS, this measurement can prove very useful not only for background rejection, since physics collisions have to happen in a very narrow window synchronous to the beam clock, but also for some physics channels with "delayed" $\gamma$ 's or vertex determination for decays with neutral particles (e.g., $H \rightarrow \gamma \gamma$ ).

The prompt current in a LAr calorimeter can thus be fully exploited on the condition that the whole electronics chain with the clock distribution and signal sampling does not degrade it. The time resolution by the electronics chain is as good as $\sigma_{T} / T=2.55 \mathrm{~ns} \cdot \mathrm{GeV} / E(\mathrm{GeV}) \oplus$ 13 ps for single cells. The time resolution measured with electrons at $70 \mathrm{GeV}$ is $\sim 70 \mathrm{ps}$.

\section{CONCLUSION}

The ATLAS electromagnetic calorimeter has concluded the construction period. At CERN, the integration of modules into the cryostats, including associated tests, is well advanced. Cold tests are expected to be performed soon. The quality in terms of performance (from test beam results) and number of defective channels found gives us good expectations for a successful operation within ATLAS. 


\section{ACKNOWLEDGMENT}

The work described in this paper is the result of many years of efforts of past and present members of the Electromagnetic (EM) Group of the ATLAS LAr collaboration. The authors would like to thank a long list of colleagues who helped in the preparation of this manuscript.

\section{REFERENCES}

[1] ATLAS Collaboration, Detector and Physics Performance Tech. Design Rep., Vol. I and II, CERN LHCC/99-14 and CERN LHCC/99-15.

[2] $\longrightarrow$ Calorimeter Performance Tech. Design Rep., CERN LHCC/96-40.

[3] - Liquid Argon Calorimeter Tech. Design Rep., CERN LHCC/96-41.

[4] RD3 Collaboration, Nucl. Instrum. Methods Phys. Res. A, vol. 309, pp. 438-449, 1991.

[5] — Nucl. Instrum. Methods Phys. Res. A, vol. 321, pp. 467-478, 1992.

[6] —-, Nucl. Instrum. Methods Phys. Res. A, vol. 325, pp. 116-128, 1993.

[7] - Nucl. Instrum. Methods Phys. Res. A, vol. 389, pp. 398-408, 1997.

[8] _- Nucl. Instrum. Methods Phys. Res. A, vol. 364, pp. 296-306, 1995.

[9] ATLAS Electromagnetic Liquid Argon Calorimeter Group, Nucl. Instrum. Methods Phys. Res. A, vol. 500, pp. 178-201, 2003.

[10] — Nucl. Instrum. Methods Phys. Res. A, vol. 500, pp. 202-231, 2003.
[11] "Construction, assembly and first tests of the ATLAS electromagnetic calorimeter barrel," Nucl. Instrum. Methods Phys. Res. A, to be published.

[12] G. Garcia et al., Nucl. Instrum. Methods Phys. Res. A, vol. 418, pp. 513-521, 1998.

[13] "Development and construction of large size signal electrodes for the ATLAS EM calorimeter," Nucl. Instrum. Methods Phys. Res. A, vol. 539, pp. 558-594, 2005.

[14] C. de la Taille, "Overview of the front-end electronics for the ATLAS LAr calorimeter," in Proc. 3rd Workshop Electronics LHC Experiments, London, U.K., Sep. 1997, Paper CERN/LHCC/97-60, pp. 186-191.

[15] W. E. Cleland and E. G. Stern, Nucl. Instrum. Methods Phys. Res. A, vol. 338, pp. 467-497, 1994.

[16] Linearity of the Response to Test Beam Electrons for EM Barrel Module P13, ATL-LARG-2004-001.

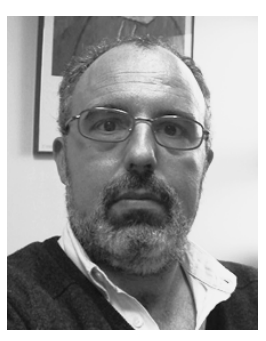

Luis Hervas received the electrical engineering degree from the Politecnic University Madrid, Spain, in 1985 and the Ph.D. degree in physics from the Universidad Autonoma de Madrid, Spain, in 1991.

$\mathrm{He}$ has participated in the ZEUS experiment at Deutsches Elektronen Synchrotron (DESY), Hamburg, Germany, and is currently working on the ATLAS experiment at CERN, Geneva Switzerland. $\mathrm{He}$ has been contributing for many years to the design, construction, installation, and commissioning of the ATLAS liquid argon calorimeter. 